\title{
¿Sensitivity of Surface Currents to Bathymetry in a Partially Mixed Estuary with Applications to Inverse Modeling
}

\author{
DORUKHAN ARDAĞ $\breve{a}^{\mathrm{a}}$ AND GREGORY WILSON ${ }^{\mathrm{a}}$ \\ ${ }^{\text {a } C o l l e g e ~ o f ~ E a r t h, ~ O c e a n, ~ a n d ~ A t m o s p h e r i c ~ S c i e n c e s, ~ O r e g o n ~ S t a t e ~ U n i v e r s i t y, ~ C o r v a l l i s, ~ O r e g o n ~}$
}

(Manuscript received 25 June 2021, in final form 29 October 2021)

\begin{abstract}
An inversion technique was tested for estimating bathymetry from observations of surface currents in a partially mixed estuary, the mouth of the Columbia River (MCR). The methodology uses an iterative ensemble-based assimilation scheme, which is found to have good skill for recovering bathymetry from observations distributed in space and time. However, the inversion skill is highly dependent on the tidal phase, location of the observations, and flow-dependent estuary dynamics. Inversion skill was found to degrade during periods of higher river discharge (up to $\sim 12000 \mathrm{~m}^{3}$ ), or low tidal amplitude, while inversion of depth-averaged velocities instead of surface velocities caused increased skill throughout the domain. These results point to dynamical limits on inversion skill, caused by changes in estuary dynamics that affect the sensitivity of surface velocities to bathymetry. An adjoint sensitivity analysis is used to visualize these effects and is combined with data-denial experiments to explore the flow-dependent inversion skill.
\end{abstract}

KEYWORDS: Data assimilation; Estuaries; Inverse methods; Model output statistics; Numerical analysis/modeling; Ocean models

\section{Introduction}

Recent technological advances in observational coastal oceanography promise increasing amounts of data from multiple sources, including drifters (Thomson 2012), satellite imagery (Horstmann et al. 2015; Ardhuin et al. 2018), X-band radar (Holman and Haller 2013; Honegger et al. 2020), and even using marine animals via biologging (Roquet et al. 2017; Chung et al. 2021). These observations offer distributed spatial coverage compared to more traditional moored and ship-based observations, but have limitations; notably, most are limited to observations near or at the water surface. Incorporating such data into numerical models can also be challenging. For example, drifter-based observations are neither synoptic nor stationary, and satellite observations are short dwell. Data assimilation and model inversion techniques offer a way to make use of such data by using it to correct model errors resulting in an overall improved model state. (Kalnay 2002; Evensen 2009).

Applications of coastal inverse modeling and data assimilation can be categorized in terms of their environmental setting (e.g., river, inlet, estuary), inversion methodology (e.g., variational, ensemble, analytical), and the variable(s) that are assumed unknown in the model (e.g., bathymetry, bottom roughness). The present work focuses on applications where the primary unknown is bathymetry, and the observations are of surface or depth-averaged currents. For a full review of past work on this topic, see Zaron (2017a). Several different methods have been developed for bathymetry inversion in rivers, e.g., Zaron et al. (2011) used a weak-constraint variational method (i.e., allowing

\footnotetext{
๑Denotes content that is immediately available upon publication as open access.
}

Corresponding author: Dorukhan Ardağ, ardagd@oregonstate.edu for errors in model forcing) to estimate bathymetry from surface currents in Haverstraw Bay, Hudson River, New York. Similarly, Almeida et al. (2018) applied a strong-constraint variational method to invert surface currents along a $95-\mathrm{km}$ reach of the Columbia River near Hanford, Washington, based on an analytical derivation of the relevant adjoint equations for nonlinear shallow water flow. Honnorat et al. (2009) developed a novel Lagrangian variational method for bathymetry inversion of surface drifter trajectories in the 2D shallow water equations. Simeonov et al. (2019) developed an analytical inversion method based on the 1D shallow water equations, including bathymetry and river discharge as unknowns, and applied it to surface current data from the Kootenai River, Idaho, and the Columbia River, Washington. Ensemble-based inversion methods have also been used for river bathymetry inversion, by Wilson and Özkan-Haller (2012) and Landon et al. (2014), for surface drifter data on the Snohomish River, Washington, and the Kootenai River, Idaho. Shifting focus to coastal ocean applications, variational methods have also been used to invert surfzone currents for bathymetry: Kurapov and Özkan-Haller (2013) investigated spatially nonuniform bathymetry inversion in a twin-test study, and Feddersen et al. (2004) inverted for bathymetry, bottom roughness, and wave forcing errors on an alongshore-uniform beach at Duck, North Carolina. Ensemble methods have been used for bathymetry inversion in the surfzone by Wilson et al. (2010, 2014), and in a well-mixed coastal inlet by Moghimi et al. (2016). And on a larger coastal scale, Zaron (2017b) used a variational method to invert tidal observations in the Sea of Okhotsk, for estimation of bathymetry and bottom roughness.

A notable common factor in all of the above bathymetry inversion studies is that the flow is shallow and vertically well mixed. Under these conditions, surface currents tend to be strongly sensitive to bathymetry, as evidenced by the success of the bathymetry inversion methods. In contrast, non-wellmixed flows (e.g., in estuaries) may be influenced by 
baroclinic effects in addition to bathymetry, and these effects may be particularly important if attempting to invert observational data taken at the water surface. An extreme possibility is that the surface flow might become essentially decoupled from bathymetry, in which case bathymetry inversion would not be possible, and indeed may not even be needed if the goal is to improve model predictions through data assimilation. To determine if and when such (in)sensitivity occurs, the present work aims to quantify surface flow sensitivity to bathymetry in a realistic baroclinic flow setting. Noting that sensitivity will change in time in response to estuary dynamics, a key goal of the study is to identify when and where sensitivity does and does not occur. A potential application would be to provide guidance for optimal time and location of surface current observations to produce the most accurate bathymetric estimate in an inverse model.

In this study, a previously validated forward model setup at a large partially mixed estuary, the mouth of the Columbia River (MCR), was utilized to create an ensemble of high-resolution model simulations (Akan et al. 2017). Outputs from the MCR model runs were used to quantify the statistical covariance between the observed and modeled variables, namely, surface current velocities $\mathbf{u}_{\text {surf }}=\left(\mathbf{u}_{\text {surf }}, \mathbf{v}_{\text {surf }}\right)$, depth-averaged current velocities $\mathbf{u}_{\text {bar }}=\left(\mathbf{u}_{\text {bar }}, \mathbf{v}_{\text {bar }}\right)$, and bathymetry $\mathbf{h}$. The covariances were then used in a least squares inversion to recover $\mathbf{h}$ from either $\mathbf{u}_{\text {surf }}$ or $\mathbf{u}_{\text {bar. }}$. It will be shown that the tidal phase, river discharge, and spatial variability of bathymetry and flow dynamics all exert significant influence on inversion skill, as determined through a series of inversion experiments and an adjoint sensitivity analysis.

The paper is organized as follows: section 2 describes the bathymetry inversion methodology and the characteristics of the study area to indicate relevant dynamics during the study period; section 3 displays the results from the simulations under multiple tidal scenarios to determine the impact of the flow dynamics on the results; and section 4 is dedicated to a discussion on adjoint sensitivities, a novel iterative approach, and an overall summary of the findings.

\section{Methodology}

\section{a. Study site and model setup}

The Columbia River is the fourth-largest river in North America with a mean discharge of $7730 \mathrm{~m}^{3}$ (Stanford et al. 2005). Bathymetric features at the MCR (Fig. 1) are highly dynamic, being subject to yearlong strong hydrodynamic forcing from a relatively high tidal range and monthly tidal variations, as well as surface gravity wave forcing that tends to promote an infilling of the estuary with sediment, counteracted by the placement of engineered jetties and annual dredging for navigational purposes. Navigational channels near the river mouth reach deeper than 30 $\mathrm{m}$ at certain parts along the estuary (Honegger et al. 2020), and dredge-spoil placements create shoals just offshore of its mouth. The MCR is classified as a partially mixed estuary with enhanced stratification (Chawla et al. 2008; Kärnä et al. 2015) caused by a high river discharge, which modulates the surface velocities and generates a buoyant plume within and offshore of the river mouth (Nash et al. 2009; Akan et al. 2017, 2018). The time period used for the present study was 0000 UTC 23 May to 0000 UTC 15 July 2014, spanning a full spring-neap tidal cycle during the spring freshet. Thus, the time period includes significant stratification due to the presence of the buoyant river discharge and nearbottom salt wedge, which are hypothesized to affect the sensitivity of surface currents to bathymetry.

A high-resolution (200-m horizontal, 40 vertical layers) nested numerical model, Coupled Ocean-Atmosphere-Wave-Sediment Transport Modeling System (COAWST) (Warner et al. 2010), was adapted for MCR simulations. This model has been used and validated previously for this region by Akan et al. (2017, 2018), whose same setup was used here with updated boundary and initial conditions for the target time period. For the present analysis COAWST was used in ROMS-only mode (i.e., neglecting the effects of surface gravity waves), and only solving for the estuary hydrodynamics (e.g., sediment transport is not considered). Initial and boundary conditions for temperature, salinity, surface water level and horizontal momentum conditions, were defined using daily averages from the West Coast Operational Modeling System (WCOFS) regional model Kurapov et al. (2011, 2017), whose domain encompasses the U.S. West Coast from California to Washington with $2-\mathrm{km}$ grid resolution. Notably, the WCOFS model grid includes a representation of the Columbia River and its discharge. Tidal constituents were obtained from the global model TPXO (Egbert and Erofeeva 2002). Atmospheric fields were imported from North American Mesoscale Forecast System (NAM), and atmospheric bulk fluxes were calculated by using Fairall et al. (1996) similar to Akan et al. (2017). Freshwater discharge and water temperature from the Columbia River was forced based on measurements by the U.S. Geological Survey (USGS) gauge 14246900. Long-term annual water temperature averages were adopted in case of gaps within the data, which happened over a 2-week span during our study period. We note that, if any of these input parameters cannot be specified exactly, they would lead to model errors. Since the present methodology assumes a perfect model, such model errors would in turn lead to errors in bathymetry inversion. Incorporating model error into the ensemble inversion framework is therefore an important avenue for future work, for example a larger ensemble could include random perturbations in unknown parameters (such as river discharge, bottom roughness, turbulence mixing diffusivity); however, this is considered beyond the scope of the present idealized study.

The skill of this MCR COAWST model has been analyzed in detail by previous studies (Akan et al. 2017, 2018), and so will be discussed only briefly here. Figure 2 compares the output of the model run with true bathymetry to observations of water surface elevation and salinity. The surface elevation time series were collected by NOAA (Hammond tidal station), and salinity time series were collected by the Center for Coastal Margin Observation and Prediction (CMOP) interdisciplinary SATURN network (Baptista et al. 2015) Jetty A and Saturn01 stations, at 6.4- and 19.5-m depth. Overall, the model captures tidal/subtidal variations in the observed variables accurately, comparable to skill seen in the previous studies. 


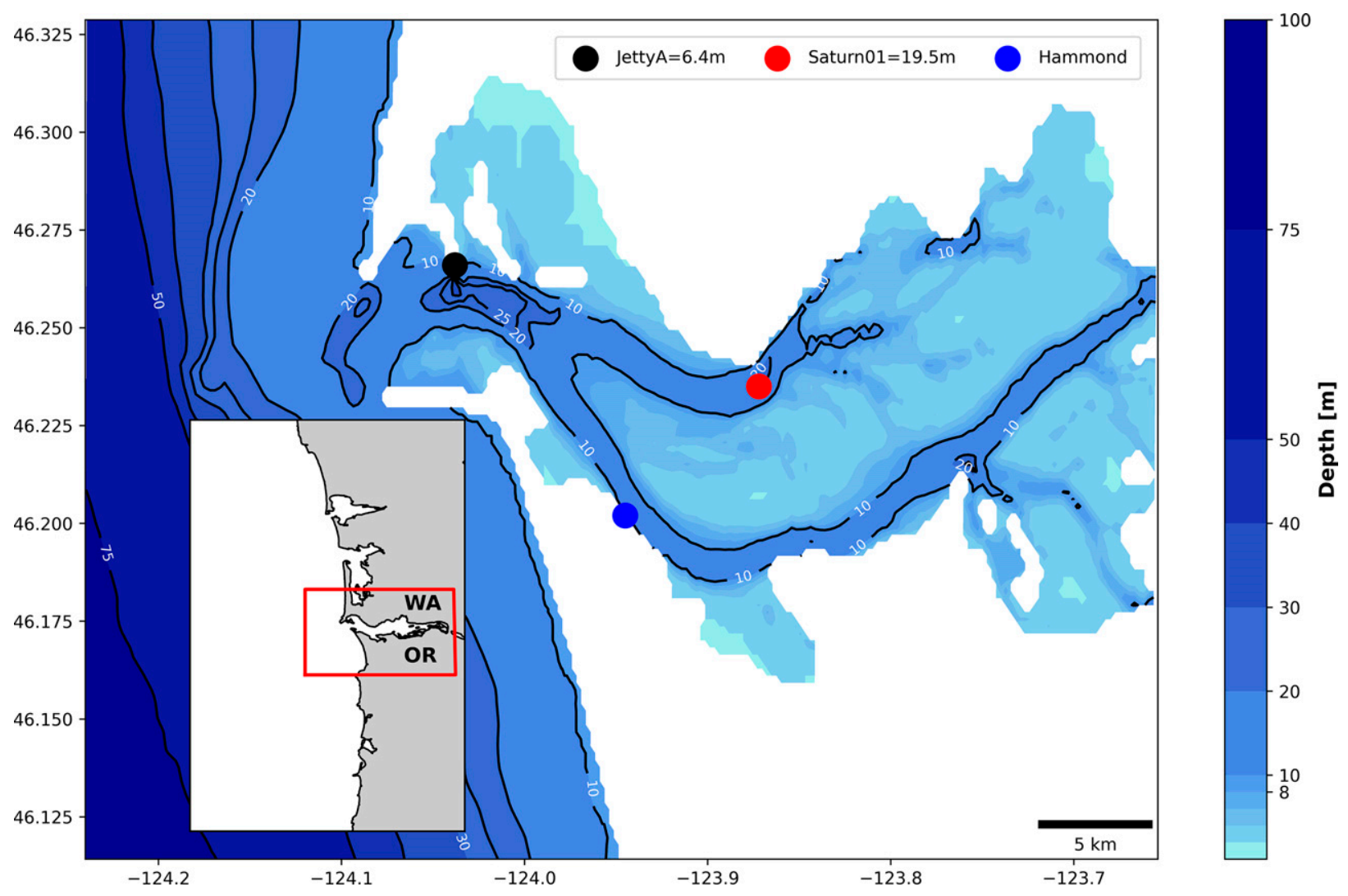

FIG. 1. Study domain and bathymetry, mouth of the Columbia River (MCR), Oregon, United States. In situ moorings used for forward model validation are marked by circles, with data provided by Coastal Margin Observation and Prediction (CMOP) stations Jetty A (6.4m depth; black circle) and Saturn01 (19.5-m depth; red circle), and NOAA Hammond tidal gauge (blue circle).

\section{b. Bathymetry inversion methods}

An ensemble-based inversion scheme was developed to estimate the spatially dependent bathymetry in the MCR based on observations of surface currents distributed in space and time. A similar inversion framework has been previously validated in a number of coastal and riverine studies (Wilson et al. 2010; Wilson and Özkan-Haller 2012; Moghimi et al. 2016), although notably has not been tested in a partially mixed estuary such as the MCR. Interested readers are referred to those earlier works for a detailed description of the inverse methodology, which is based on a state-augmented ensemble-based parameter estimation (Evensen 2009). Briefly, the inverse problem involves a set of observations of flow velocity $\mathbf{u}$ located at $K$ observation points in the model domain, which are to be inverted for the model bathymetry $\mathbf{h}$, an unknown parameter of size $M \times 1$ (where $M$ is the number of model grid points). Extension to additional observed variables (other than u) or other parameters (other than h) is straightforward. The inversion seeks to minimize a least squares cost function,

$$
\mathcal{J}[\mathbf{h}]=\mathcal{J}_{\text {obs }}+\mathcal{J}_{\text {bg }}
$$

where

$$
\mathcal{J}_{\text {obs }}[\mathbf{h}]=(\mathbf{d}-\mathbf{M h})^{\mathrm{T}} \mathbf{C}_{d}^{-1}(\mathbf{d}-\mathbf{M h}),
$$

and

$$
\mathcal{J}_{\text {bg }}[\mathbf{h}]=\left(\mathbf{h}-\mathbf{h}^{f}\right)^{\mathrm{T}} \mathbf{C}_{\mathbf{h h}}^{-1}\left(\mathbf{h}-\mathbf{h}^{f}\right)
$$

Here, the $K \times 1$ vector $\mathbf{d}$ contains the measurements, and $\mathbf{M}$ is a $K \times M$ matrix which serves to extract the corresponding model predictions at the measurement locations, given $\mathbf{h}$. Note that in general the relationship between $\mathbf{u}$ and $\mathbf{h}$ is nonlinear, so the matrix $\mathbf{M}$ implies this relationship has been linearized; the linearization is based around a prespecified prior (i.e., background) bathymetry $\mathbf{h}^{f}$, whose uncertainty is specified by a covariance matrix $\mathbf{C}_{\mathbf{h h}}$. The two terms in the cost function, $\mathcal{J}_{\text {bg }}$ and $\mathcal{J}_{\text {obs }}$, serve to penalize departures from this prespecified prior, and from the observations, respectively. The requirement of a prior is to constrain the underdetermined inverse problem (i.e., the number of observations is assumed small compared to the dimension of the unknown bathymetry), and it provides a reference solution around which the model dynamics are linearized. Finally, algebraic 

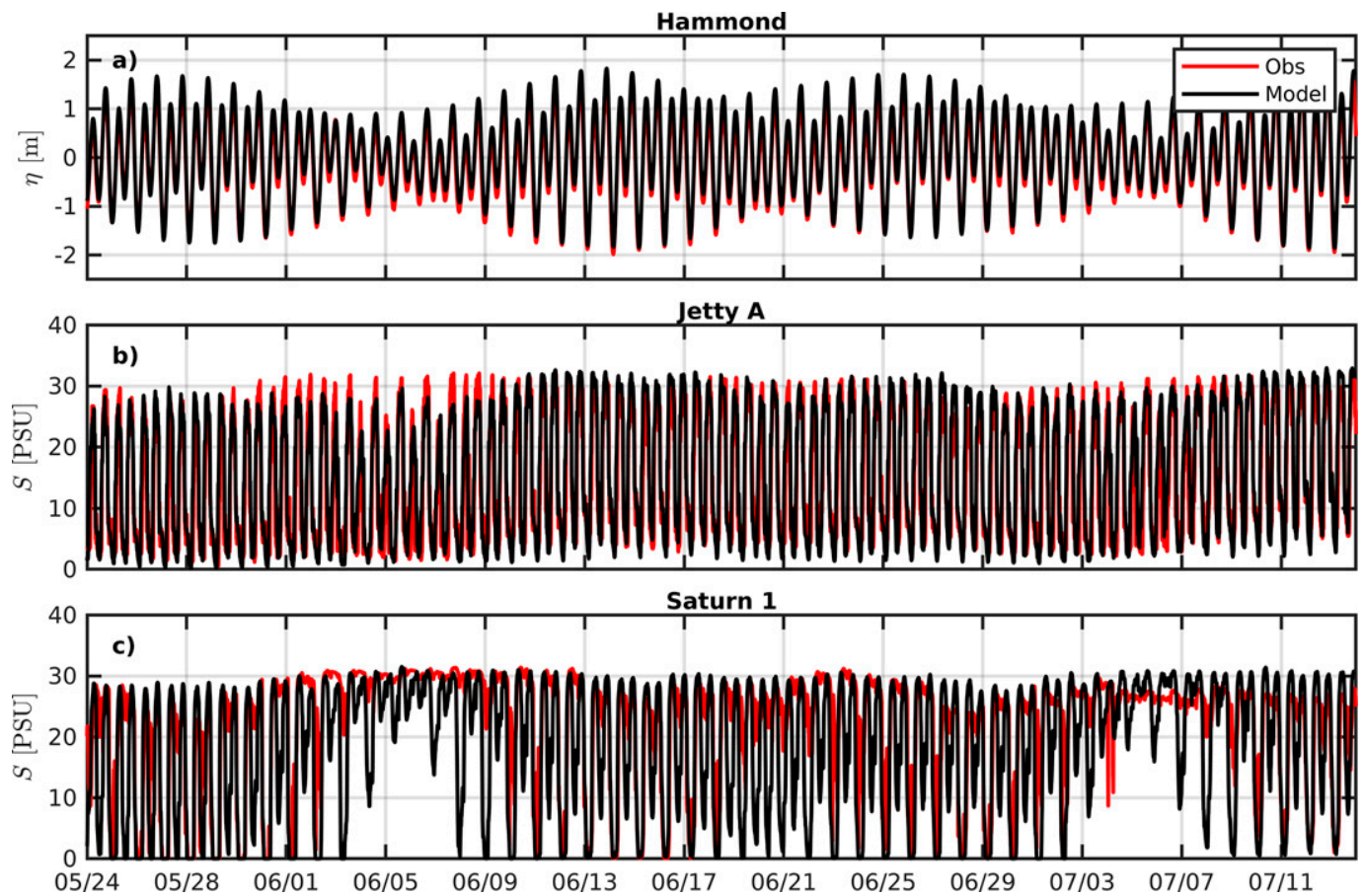

FIG. 2. Comparisons between model (black lines) and observations (red lines) at in situ moorings shown in Fig. 1, for the time period 24 May to 15 Jul 2013 (times are in UTC). The variables compared are (a) sea surface elevation $(\eta)$ at Hammond tidal gauge, (b) salinity $(S)$ at Jetty A, and (c) salinity $(S)$ at Saturn01.

minimization of Eq. (1) results in a solution for the most likely bathymetry, or "analysis,"

$$
\mathbf{h}^{a}=\mathbf{h}^{f}+\mathbf{C}_{\mathbf{h h}} \mathbf{M}^{\mathrm{T}}\left(\mathbf{M} \mathbf{C}_{\mathbf{h h}} \mathbf{M}^{\mathrm{T}}+\mathbf{C}_{\mathbf{d}}\right)^{-1}\left(\mathbf{d}-\mathbf{M h}^{f}\right) .
$$

For the present work, the prior bathymetry was chosen to conform to the known MCR coastline, and has a realistic spatially averaged depth, but lacks any features such as channels and shoals (see section 2c). The prior covariance $\mathbf{C}_{\mathrm{hh}}$ is assumed to have a Gaussian form

$$
\mathbf{C}_{\mathrm{hh}}(\Delta i, \Delta j)=\sigma_{\mathbf{h}}^{2} \exp \left[-3 \frac{\Delta i^{2}}{L_{i}^{2}}-3 \frac{\Delta j^{2}}{L_{j}^{2}}\right]
$$

where $\Delta i$ and $\Delta j$ are separation distances; $\sigma_{h}$ represents the amplitude of uncertainty in the prior $\mathbf{h}^{f}$, and can be spatially varying; and $L_{i}$ and $L_{j}$ are spatial scales in the $i$ and $j$ model grid directions, respectively. The factor 3 in this equation is included so that $L_{i}$ and $L_{j}$ correspond to the separation distances at which correlation is reduced to less than 5\%. Following Moghimi et al. (2016), we compute this prior covariance on a curvilinear model grid that incorporates the expected shapes of bathymetric features, see section $2 c$.

To solve Eq. (4), the terms $\mathbf{C}_{\mathrm{hh}} \mathbf{M}^{\mathrm{T}}, \mathbf{M} \mathbf{C}_{\mathrm{hh}} \mathbf{M}^{\mathrm{T}}$, and $\mathbf{M h}^{f}$ are approximated based on an ensemble of realizations of model runs, with bathymetry realizations drawn from the specified prior distribution. In the present work a total of $N=300$ realizations were used, which are passed through the numerical model (section 2a) to compute corresponding ensembles for $\mathbf{u}$.
The ensemble is then used to approximate $\mathbf{C}_{\mathrm{hh}} \mathbf{M}^{\mathrm{T}}$ and $\mathbf{M} \mathbf{C}_{\mathrm{hh}} \mathbf{M}^{\mathrm{T}}$ based on the sample covariances between bathymetry and the ensemble of $\mathbf{u}$, while the ensemble mean of $\mathbf{u}$ is measured to obtain $\mathbf{M h}^{f}$. Observational data are assumed to have identical and independent error statistics, so that the observation covariance $\mathbf{C}_{\mathbf{d}}$ is a diagonal matrix with constant variance $R$. The magnitude of $R$ relative to $\mathbf{C}_{\mathbf{u u}}$ effectively determines the amount of weight given to observations, with larger $R$ corresponding to less observation impact. In all cases, the method of Hamill et al. (2001) and Wilson and Özkan-Haller (2012) was used to remove spurious nonlocal correlations when computing sample covariances; a localization length of $3 \mathrm{~km}$ was chosen, approximately equal to the MCR channel width.

Equation (4) assumes linearized model dynamics about a prior state with $\mathbf{h}=\mathbf{h}^{f}$, which can lead to errors because the relationship between $\mathbf{h}$ and $\mathbf{u}$ is nonlinear. Therefore, in the present work a new approach is considered in which the inversion is iterated [e.g., see Kalnay and Yang (2010) for a discussion of a similar method]. For each iteration, the analysis $\mathbf{h}^{a}$ from the previous iteration is used as the prior $\mathbf{h}^{f}$. Iterations are continued until changes in $\mathcal{J}_{\text {obs }}$ drop below $15 \%$ between successive iterations. Strictly speaking, the iterative approach violates the statistical justification that usually underlies a least squares inversion: That $\mathbf{h}^{f}$ and $\mathbf{C}_{\mathbf{h h}}$ represent the mean and covariance of a Gaussian-distributed (unknown) true bathymetry, thus defining a prior distribution in the Bayesian sense. The iterative scheme updates the prior with each iteration, based on observational data, hence repeated application of the inverse step would tend to overfit the measurements if a 


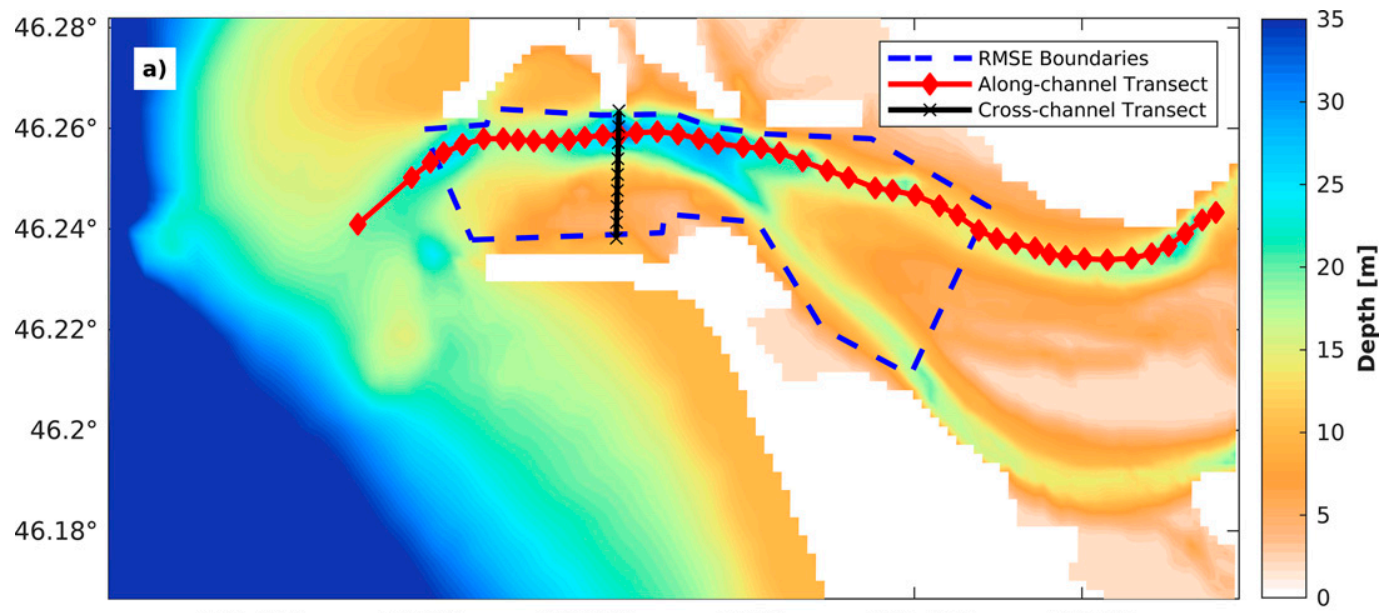

$\begin{array}{llllll}-124.15^{\circ} & -124.1^{\circ} & -124.05^{\circ} & -124^{\circ} & -123.95^{\circ} & -123.9^{\circ}\end{array}$

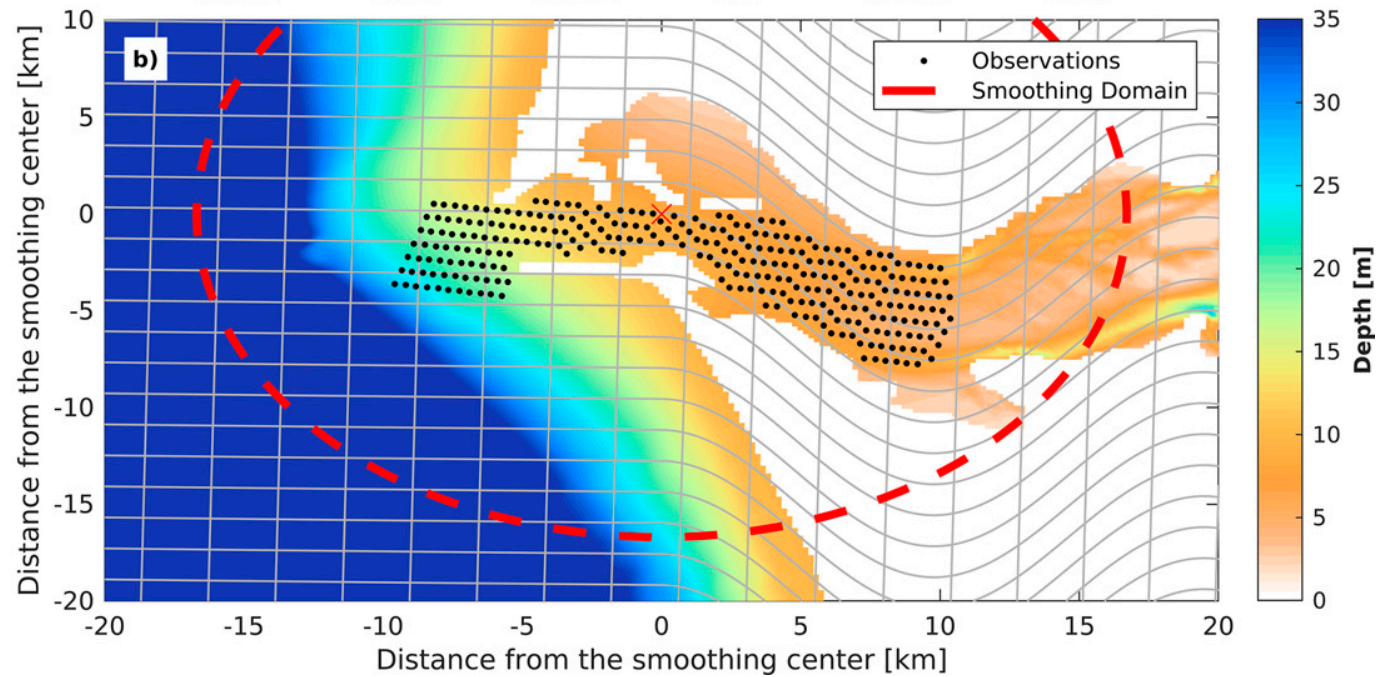

FIG. 3. (a) MCR true bathymetry and (b) prior bathymetry, plotted as depth below mean tide level. Dashed blue lines in (a) indicate subdomains used for comparisons of inversion skill (RMSE). Red line with diamonds in (a) is the alongchannel transect used for verification in the north navigational channel, and black line with crosses is the cross-channel transect cutting through the deepest part of the MCR $(\sim 35 \mathrm{~m})$. Gray grid lines in (b) show the curvilinear grid used for calculating the ensemble of perturbations to bathymetry, Eq. (5), where the grid spacing corresponds to perturbation length scales $L_{i}$ and $L_{i}$. Perturbations are smoothly tapered to zero beyond the region bounded by the red dashed circle in (b) to avoid boundary effects, while within this region the perturbations have uniform standard deviation equal to $\sigma_{\mathbf{h}}$.

Bayesian interpretation is accepted. In the depth-inversion applications such as the one considered here however, there is usually limited information with which to specify the prior. Instead, the prior serves mainly to (i) regularize the inversion and avoid nonphysical corrections and (ii) provide a base state around which the model dynamics are linearized. In that sense, the iterative scheme can be viewed as a regularized gradientdescent-type method, which seeks to minimize model-data misfit while avoiding nonphysical corrections. The choice of $\sigma_{\mathbf{h}}$ and $R$ in this scheme effectively determine the step size for each gradient descent step. For the present work, values of $\sigma_{\mathbf{h}}=2 \mathrm{~m}$ and $R=0.5^{2} \mathrm{~m}^{2} \mathrm{~s}^{-2}$ were found to give accurate results for the iterative tests. For single step or "snapshot" inversions, and adjoint sensitivity calculations, $\sigma_{\mathbf{h}}=5 \mathrm{~m}$ and
$R=0.5^{2} \mathrm{~m}^{2} \mathrm{~s}^{-2}$ were employed instead, since iterations were not practical for these tests due to computational limitations. The use of a larger $\sigma_{\mathbf{h}}$ leads to larger corrections in a single iteration, at the possible expense of errors for highly nonlinear inversions. The overall conclusions of the study were not affected by changes in the value of $\sigma_{\mathbf{h}}$, but the optimal choice for this parameter remains a subject for future work.

\section{c. Inversion test cases}

The inverse scheme was evaluated using identical-twin tests, meaning observational "data" were produced by the numerical model using the true MCR bathymetry. This approach guarantees that model error/bias is not a factor in the inverse results, hence only the inverse scheme itself is being verified. Hereafter, 
TABLE 1. Time period of study, separated into periods of spring and neap tide conditions.

\begin{tabular}{lccc}
\hline \hline Tide condition & Beginning & End & Time steps (1.5-h intervals) \\
\hline Spring tide & 0728 UTC 27 May & 2228 UTC 27 May & 11 \\
Neap tide & 2040 UTC 3 Jun & 1910 UTC 4 Jun & 16 \\
\hline
\end{tabular}

we will refer to the results from the reference simulation with true MCR bathymetry as "truth." Synoptic measurements of surface velocity were sampled from the true model run every $1.5 \mathrm{~h}$, on a regular coarse grid ( $\sim 700$-m resolution) consisting of 288 observation points covering the river mouth, see Fig. 3. These observations spanned a total of 27 time steps (1.5 days), including three tidal cycles, with 11 and 16 time steps during spring and neap tide conditions, respectively, as summarized in Table 1 . Finally, variations on the observation scheme and model setup were employed to investigate flow-dependent inverse model skill, as follows:

- Time dependence of inverse model skill was quantified by assimilating observations separately for each 1.5-h time step, treating each time step as its own independent dataset.

- Spatially dependent inverse model skill was quantified by further subsampling the observations into specific subregions of the model domain: one region near to the MCR "mouth" $\left(46.2480^{\circ} \mathrm{N}, 124.0832^{\circ} \mathrm{W}\right)$, and a second located in the "estuary" $\left(46.2523^{\circ} \mathrm{N}, 124.0119^{\circ} \mathrm{W}\right)$, as shown in Fig. 4.

- Effects of freshwater river discharge were tested by disabling or enabling the river forcing in the model (both truth, prior, and ensemble).

- Baroclinic effects were tested by comparing assimilation of surface currents versus depth-averaged (barotropic) currents.

The inverse model requires initialization of the prior bathymetry $\mathbf{h}^{f}$, which for the present study was based on a highly smoothed version of the true MCR bathymetry. The true bathymetry was smoothed within a circle that has $\sim 20-\mathrm{km}$ radius using a 2D Gaussian filter (Fig. 3b illustrates the smoothing domain). The prior covariance was initialized with constant $\sigma_{\mathbf{h}}$ in the vicinity of the river mouth, decaying to zero at the model boundaries. As stated previously, the spatial covariance was defined on a curvilinear grid that approximately conforms to the MCR coastline, such that the ensemble perturbations to bathymetry are roughly aligned with the overall MCR geometry. A depiction of the curvilinear grid and the specified initial prior is shown in Fig. 3b. After initialization, the initial prior mean bathymetry is updated iteratively, with a new ensemble of perturbations for each iteration sampled from the same covariance matrix $\mathbf{C}_{\mathbf{h h}}$ (unchanged for each iteration).

\section{d. Adjoint sensitivity analysis}

The matrix $\mathbf{C}_{\mathbf{h h}} \mathbf{M}^{\mathrm{T}}$ in Eq. (4) mathematically represents the adjoint sensitivity of $\mathbf{h}$ to the observations of $\mathbf{u}$, after convolution with the prior $\mathbf{h}$-covariance $\mathbf{C}_{\mathbf{h h}}$. In other words, it is a measure of the sensitivity of surface current $\mathbf{u}$ to perturbations in depth $\mathbf{h}$, where the latter is constrained by the prior distribution for $\mathbf{h}$ (defined by $\mathbf{h}^{f}, \mathbf{C}_{\mathbf{h h}}$ ). Considering a single grid point $i$, the sensitivity of an observation $u_{i}$ to perturbations of $h_{i}$ at the same location is given by the diagonal entry of the sample covariance between $\mathbf{h}$ and $\mathbf{u}$ [i.e., $\left(\mathbf{C}_{\mathbf{h u}}\right)_{i i}$ ]. Normalizing, $m_{i}=\left(\mathbf{C}_{\mathbf{h u}}\right)_{i i} /\left(\mathbf{C}_{\mathbf{h h}}\right)_{i i}$ can also be interpreted as the slope of a regression fit to the ensemble of $u_{i}$ versus $h_{i}$, (i.e., it is a statistical estimate of $\left.\partial u_{i} / \partial h_{i}\right)$. This is illustrated graphically in Fig. 6, which shows an ensemble of $u_{i}$ versus $h_{i}$ for two model grid points $i$, along with corresponding regression fit lines. The metric $m_{i}$ will be used to quantify the sensitivity of surface current to depth perturbations, with larger magnitudes of $m_{i}$ indicating greater sensitivity.

\section{Results}

\section{a. Inverse model validation}

In an identical-twin test (as in the present study), it is expected that the inverse method should be capable of recovering bathymetry with a high degree of accuracy, provided the

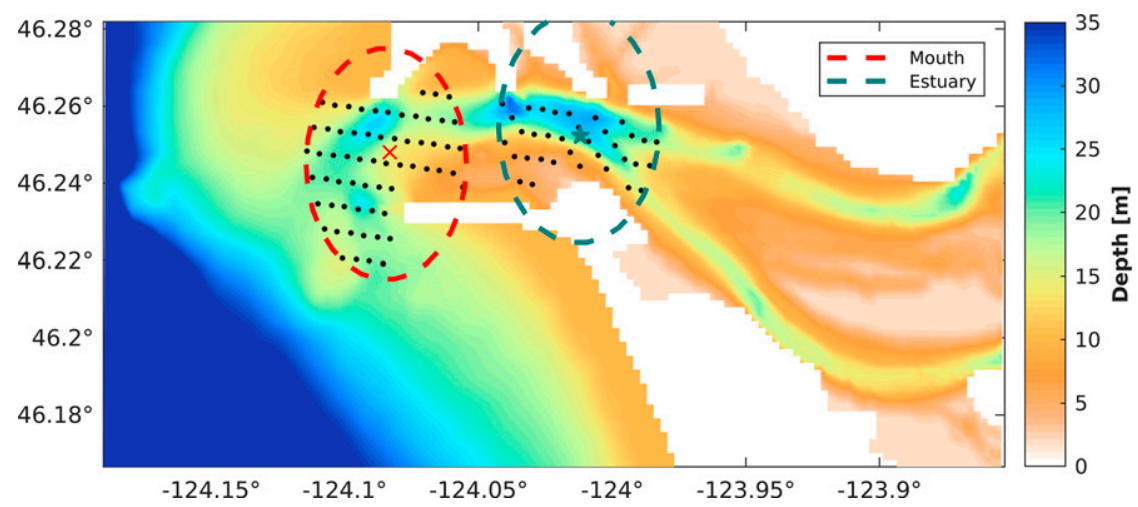

FIG. 4. Subdomains used for testing spatial dependence of inversion results: "mouth" subdomain is centered around red cross, and "estuary" subdomain is centered around teal star. Observation points for each subdomain are shown as black dots. 
data being inverted are sufficiently sensitive to bathymetry. By way of example, we begin by showing results where the data consist of 11 time steps of surface currents measured during spring tide (total of 3096 observation points). Note this inversion assimilates the observations in a single batch, rather than sequentially in time. Data from neap tide were also considered, but are excluded from this example case because they were found to have weak sensitivity to bathymetry (see section $3 \mathrm{c}$ ). This test case therefore illustrates the "near-optimal" inversion scenario, which should be compared to suboptimal cases examined in more detail later.

The iterative assimilation scheme converged after five steps, ending with a root-mean-square error (RMSE) of $2.68 \mathrm{~m}$. Figure 5a shows the true bathymetry along with the observation and transect locations, while Fig. 5b shows the final posterior results. It can be seen that the corrections capture the general shape of the prominent navigational channel, and the dual channels upstream of the river mouth. Figures $5 \mathrm{c}$ and $5 \mathrm{~d}$ show the inversion result on two representative transects: the first spanning across the MCR mouth at the location of Jetty A, which coincides with the deepest part of the navigational channel and the second being a 20-km transect following the navigational channel in the northern half of the MCR. Results are plotted for each iterative step, showing a steady convergence toward the true bathymetry. The prior profile has a 7.64-m RMSE within the section of the domain marked by the blue dashed line in Fig. 3a. Some shortcomings of the method can also be seen from Figs. $5 \mathrm{c}$ and 5d; the full depths of the channels were unable to be captured, which may be due to diminishing corrections as the number of iterations is increased. These underestimations of deep channels were not significantly improved by subsequent iterations, suggesting either a limitation in either the inverse solver or a more basic limitation of the inverse problem. Similar underestimations have been observed for river bathymetry inversion (Wilson and Özkan-Haller 2012; Landon et al. 2014; Almeida et al. 2018). Most notably, Almeida et al. (2018) reported underestimations of the largest river depths in their study using an iterative variational inverse solver, suggesting the effect is not specific to the ensemble inverse method. In all of the present applications, the iterations had $\mathcal{J}_{\text {obs }}$ decreasing with each iteration, and reached the defined convergence criteria in fewer than six iterations. Last, it is worth noting that smaller values of $R$, or larger values of $\sigma_{\mathbf{h}}$, led to an even closer fit to the true bathymetry. While not surprising for an identical-twin test, this suggests that in practical applications the inversion accuracy would be limited mostly by the number and quality of observational data, not by the ability to invert the model dynamics. Overall, these results suggest the inverse method accurately resolves the nonlinear relationship between surface currents and bathymetry in optimal conditions when the surface currents are particularly sensitive to changes in bathymetry.

\section{b. Flow-dependent adjoint sensitivity}

Having established the validity of the inverse model scheme, we now turn to an analysis of the flow-dependent sensitivity of surface currents to bathymetry. It is anticipated that surface currents will provide differing levels of information on bathymetry, depending on the three-dimensional time-varying ocean state. To test this idea, local adjoint sensitivities were calculated for each observation time step. As a representative example, Fig. 6 shows adjoint sensitivities during spring time step 4 (one of the higher-skill inversions as will be shown later in section $3 c$ ), for observations of the east-west component of surface current u. Inset scatterplots show examples to illustrate how the adjoint sensitivity is calculated (see section $2 \mathrm{~d}$ ), by comparing the ensemble of $\mathbf{u}$ versus $\mathbf{h}$ (blue dots), along with the measured value (pink star), and prior (black star), at locations marked by the red cross and teal star on the full map. As explained in section $2 \mathrm{~d}$, the adjoint sensitivity is simply the slope of a regression fit line to the ensemble, and the posterior bathymetry [Eq. (4)] is an extrapolation of this linearized fit (to good approximation, neglecting possible contributions from other nearby observations). Regions with low adjoint sensitivity are expected to contribute minimally to bathymetry inversions, potentially leading to lower inversion skill.

The adjoint sensitivity to surface current was found to vary over time, and differed for the east-west versus north-south component of current. Figure 7 shows representative examples with adjoint sensitivity vectors $\mathcal{A}$ plotted over the bottom layer salinity to show the geometry of the salt wedge. The magnitude of $\mathcal{A}$ represents the overall sensitivity of surface currents to local bathymetry (i.e., the change in current magnitude that would be expected for a unit change in water depth). The direction of $\mathcal{A}$ shows the relative sensitivity for each component of velocity. To help illustrate the dependence on ebb versus flood tides, the sign of $\mathcal{A}$ has been negated for ebb tides (for which $\mathbf{u}$ is predominately negative). The results show that, despite being on different model days and monthly tidal phases, adjoint sensitivity is primarily a function of tidal phase. During ebb tides (Figs. 7a,b), the flow is most sensitive to bathymetry over a narrow region of the river mouth, coinciding with the deepest parts of the main navigational channel. During flood tides, the flow sensitivity extends much farther up the estuary. This analysis was also repeated for the posterior bathymetry shown in Fig. 5, with similar results (not shown). In that case, $\mathcal{A}$ was found to be largest over the navigational channels present in the bathymetry, indicating a tendency for higher sensitivity associated with channelized bathymetric features or constrictions.

\section{c. Flow-dependent inverse results}

The preceding adjoint sensitivity analysis suggests surface currents are only sensitive to bathymetry in certain regions of the MCR, which vary depending on tidal phase. To further investigate this effect, inversions were performed on single-time sets of observations spaced $1.5 \mathrm{~h}$ apart. A total of 27 time points were considered, spanning the full simulation period. These individual "snapshot" inversions did not use iterations, due to computational restrictions. However, results in section 3a suggest that the corrections obtained after one iteration are generally reinforced by subsequent iterations, for example, increasing the magnitude of the navigational channel but not its position or shape. Therefore, we expect the relative skill between different time steps 

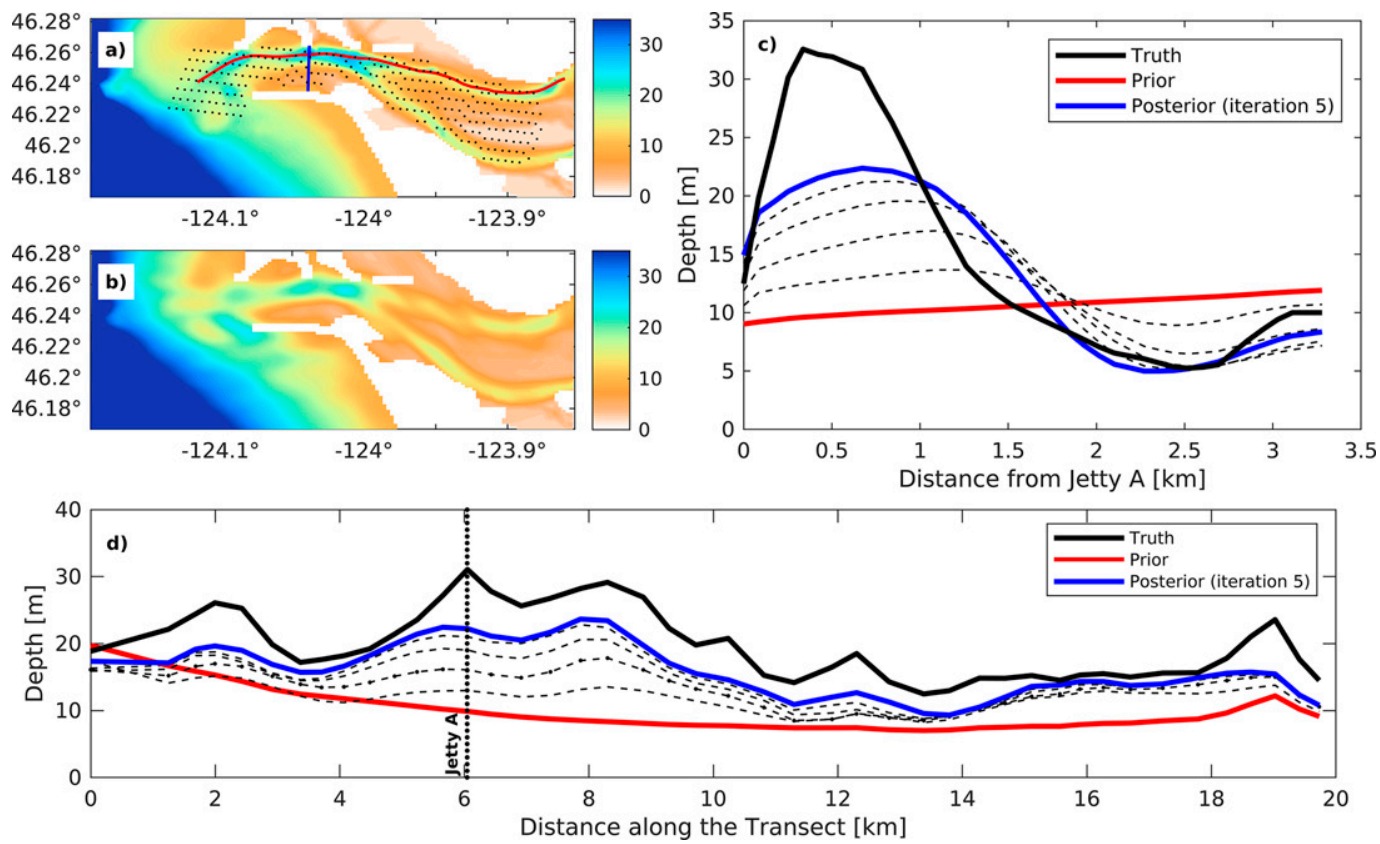

FIG. 5. Inverted bathymetry comparisons for the iterative inversion of 11 time steps during spring tide: (a) true bathymetry, observation locations, and the transect locations; (b) posterior (iteration 5) using $\mathbf{u}_{\text {surf }}$; (c) cross-channel transect comparison [see blue line in (a)]; and (d) along-channel transect comparison [see red line in (a)]. Initial prior bathymetry is shown as red line, true bathymetry is shown as black line, and results are shown for each of five iterations ending in thick blue line. Convergence after five iterations was determined based on incremental changes in $\mathcal{J}_{\text {obs }}$ as described in text.

would not change with subsequent iterations, and this relative skill is used to intercompare the results for different time steps.

Figure 8 shows the single-time inversion result with the lowest RMSE, spring time step 4 (ebb conditions). Figure $8 \mathrm{~b}$ shows the overall estimated bathymetry, which resolves much of the navigational channel in the northern side of the river mouth, and parts of the two branched channels that extend farther up the estuary. Figures $8 \mathrm{c}$ and $8 \mathrm{~d}$ show the results

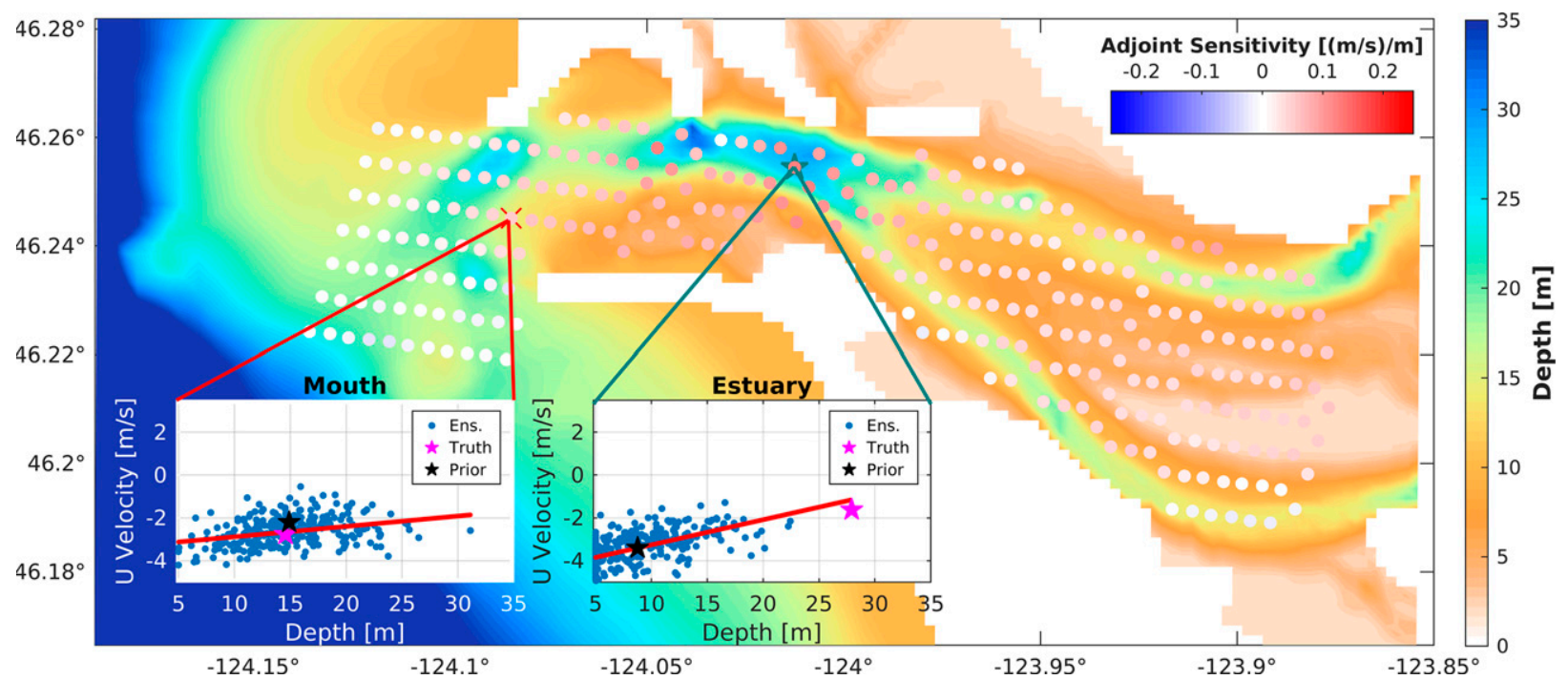

FIG. 6. Adjoint sensitivity analysis during spring tide time step 4 (ebb tide). Each colored point in main plot shows sensitivity of east-west surface currents to local bathymetry, in units of $\mathrm{m} \mathrm{s}^{-1} \mathrm{~m}^{-1}$. Insets show examples of the ensemble (blue dots), true (magenta star), and prior (black star) east-west velocities plotted vs corresponding ensemble depth values at the mouth (red cross) and the estuary (teal star); the slope of the red line corresponds to adjoint sensitivity, defined as $m_{i}$ in text. 

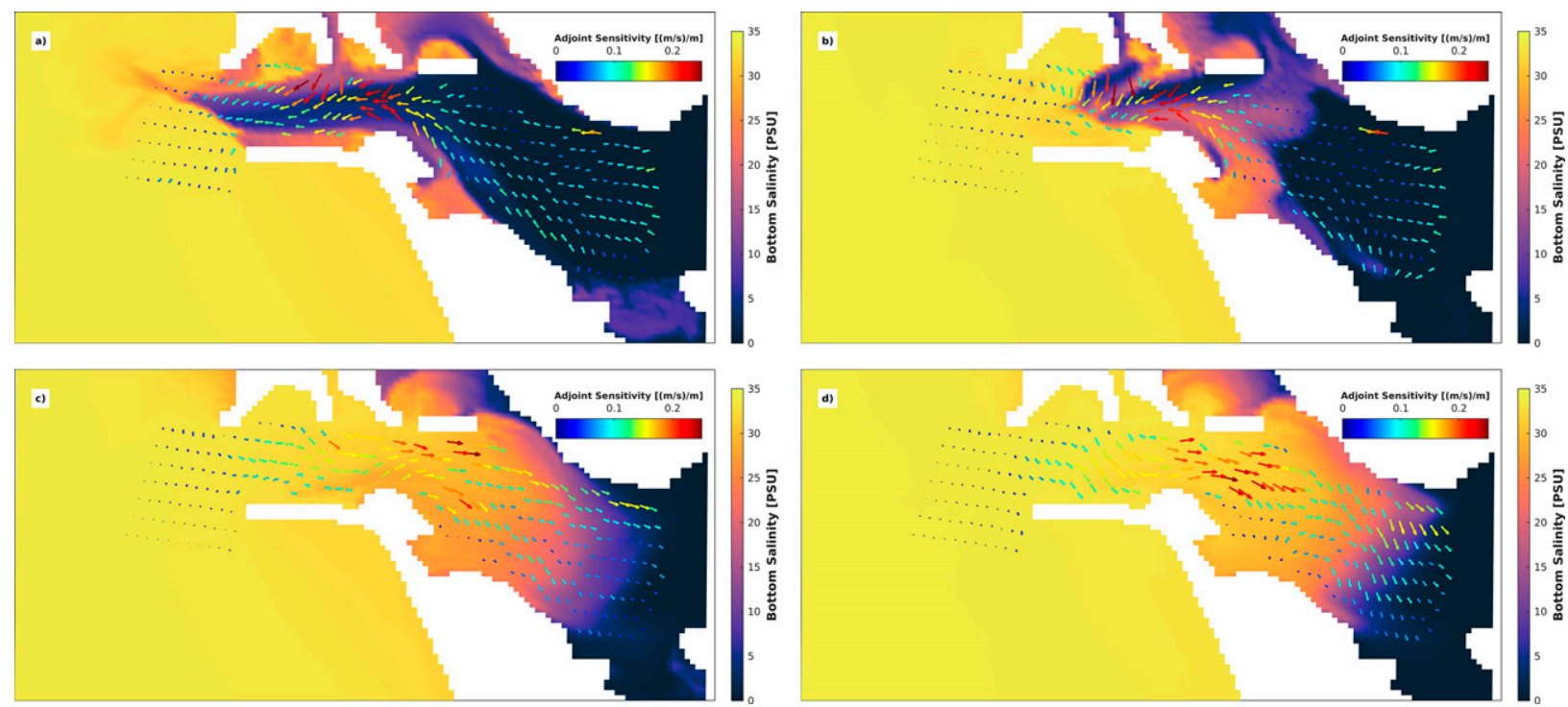

FIG. 7. Adjoint vectors $\mathcal{A}$ calculated for (a) spring ebb tide (time step 4), (b) neap ebb tide (time step 14), (c) spring flood tide (time step 9), and (d) neap flood tide (time step 4).

along transects, where red lines indicate the prior, blue lines are the posterior, and black lines are the true bathymetry. Note that in Fig. 8c (cross-channel transect) the difference between the true and prior bathymetries changes sign between the north and the south part of the channel. In the north section, the channel depth is overall underpredicted by approximately $20 \mathrm{~m}$, while in the south section the bathymetry needs to become shallower and the method is able to capture this to a certain extent. The position of the navigation channel was also mispredicted, being approximately $1 \mathrm{~km}$ to the south of its true location. These results are similar along most of the river mouth, between 6 and $12 \mathrm{~km}$-coinciding with areas of high adjoint sensitivity as shown in Fig. 7a for the same time step. Outside of this region surface velocities evidently provided little to no information on bathymetry, resulting in negligible corrections to the prior. The results
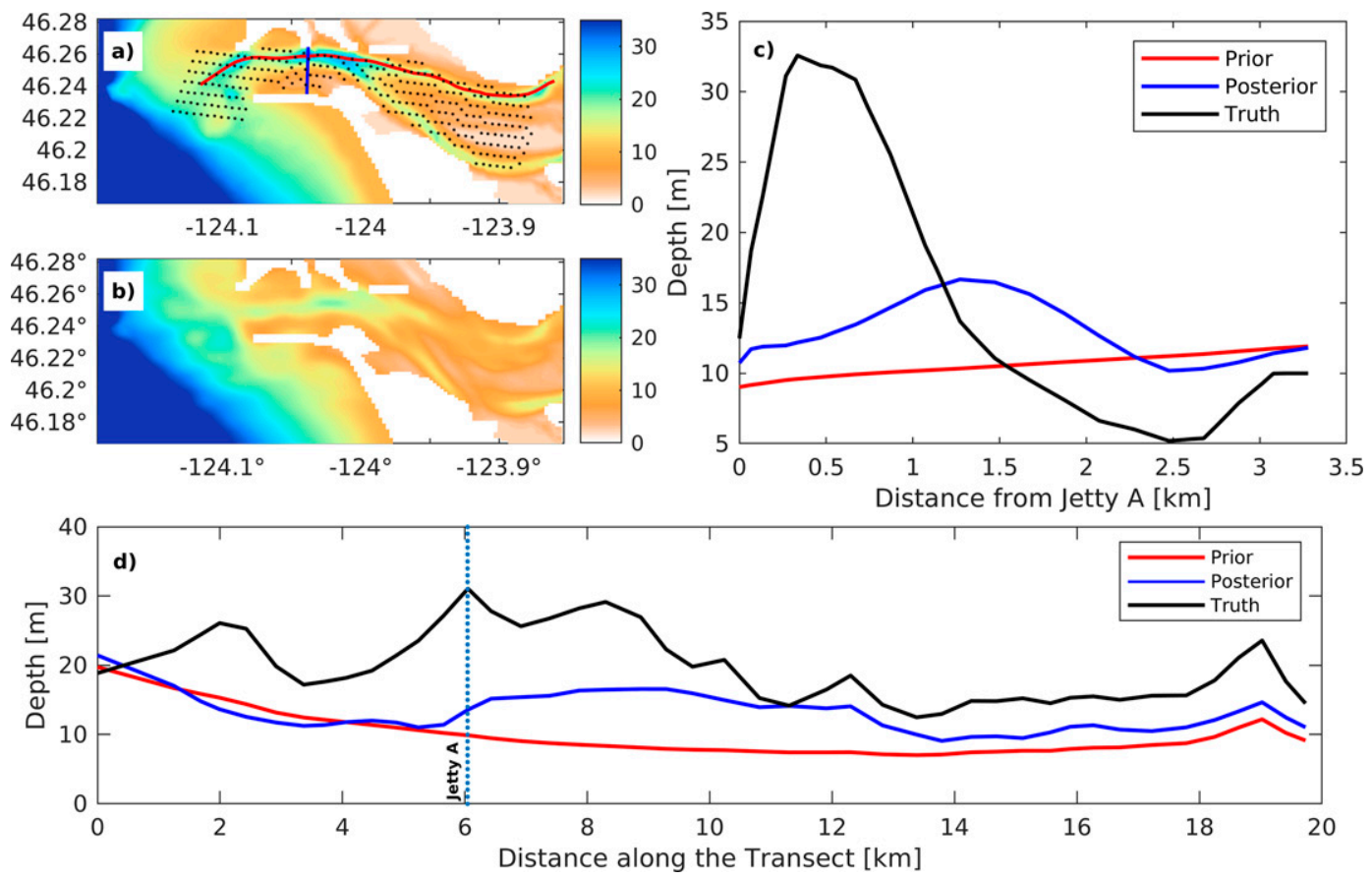

FIG. 8. As in Fig. 5, but for inversion output for spring ebb tide, single time step 4, 1158 UTC 27 May. 

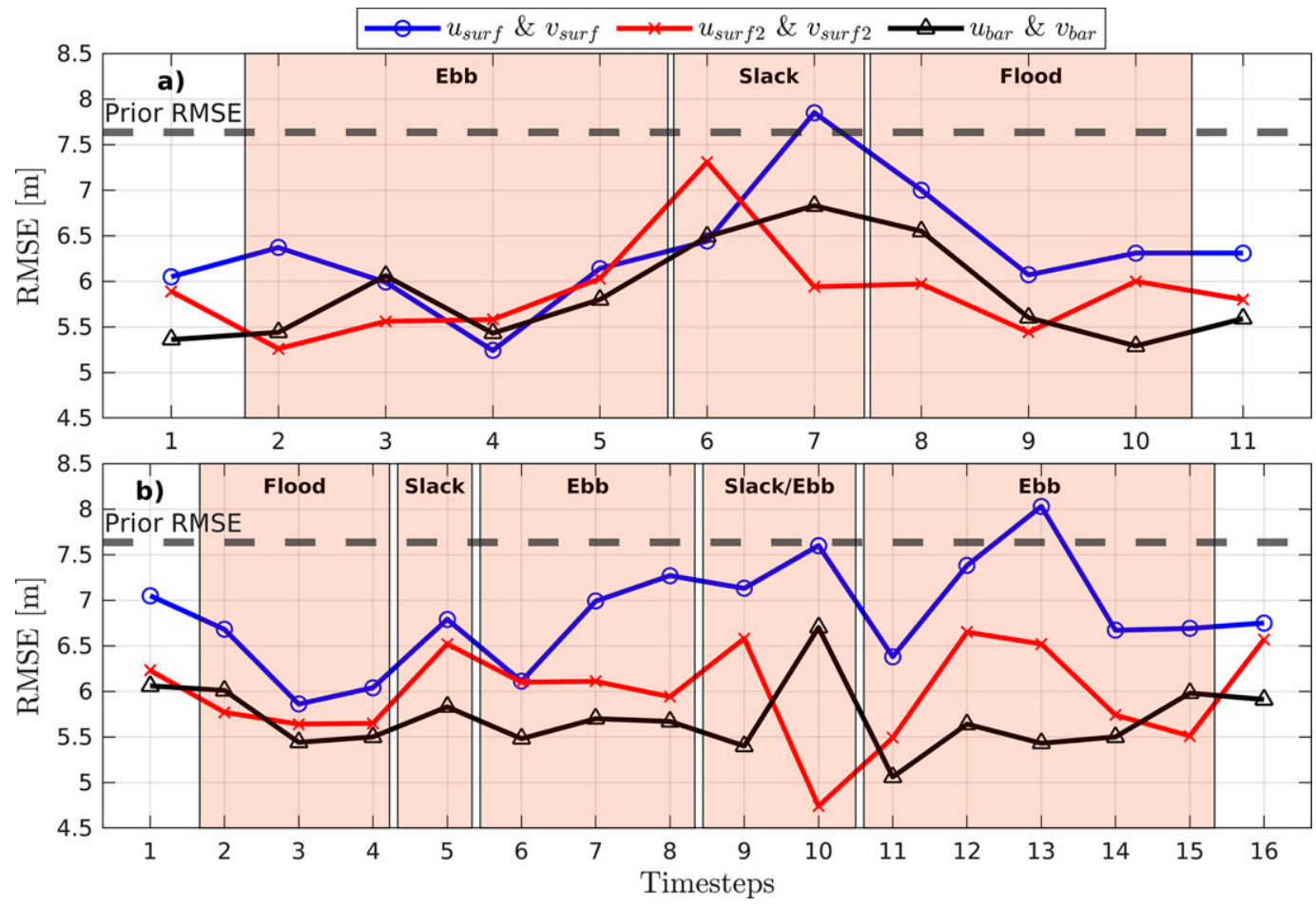

FIG. 9. Inverted bathymetry RMSE for single-time inversions during (a) spring and (b) neap tides, within the blue dashed region shown in Fig. 3. Results are shown for three cases: assimilating $\mathbf{u}_{\text {surf }}$ (blue), assimilating $\mathbf{u}_{\text {bar }}$ (black), and assimilating $\mathbf{u}_{\text {surf }}$ (red), but with river discharge excluded from model. Horizontal dashed lines show the prior RMSE; i.e., inversions that exceed this RMSE have negative skill.

were not significantly changed by increasing the density of the observational array (not shown). Based on the results shown in section $3 \mathrm{a}$, further iterations would tend to improve the results where bathymetry is under/overpredicted, while the predicted position of bathymetric features would likely not be improved by iteration.

Similar comparisons were carried out for the rest of the individual time steps. The time-dependent RMSE is shown in Fig. 9 (blue lines), evaluated for the region marked by dashed blue line in Fig. 3. The figure has been split into spring and neap tide conditions (Figs. 9a and 9b, respectively), and the timing of ebb- or flood-tidal phases are labeled for each time period as a qualitative visual aid (with the caveat that the transition between ebb and flood tide is a spatially dependent process that does not occur instantaneously). The prior RMSE (gray dashed line) is also included as a baseline reference, i.e., results with RMSE below this prior value can be interpreted as having positive skill. Overall, the results show that single-time surface velocity inversions (blue lines) tend to be more accurate during spring tides compared to neap tides, which suggests the lower mixing associated with neap tide leads to a less accurate inversion. Similarly, inversion skill tends to be maximized during high-flow conditions (peak ebb or flood tide), and is reduced during slack conditions.

Next, inversions were repeated but with river discharge turned off in the model (in the truth model run as well as the ensemble), with resulting RMSE shown as red lines in Fig. 9.
These results show a similar time-dependent RMSE trend to those with river discharge included (blue lines), except inversion skill is improved by approximately $10 \%-15 \%$ for nearly all time steps. Larger increases in skill are observed for neap tides (Fig. 9b), possibly because of a relatively larger influence of river discharge during periods of low tidal amplitude. The presence of river discharge also altered the phasing of tide in the estuary, such that the timing of ebb and flood conditions is slightly changed - most notably, slack tide appears earlier in the no-river runs during spring tide (Fig. 9a, time steps 6-7). Overall it can be concluded that the absence of river discharge consistently led to an increased inversion skill.

Inversions were also tested in which the depth-averaged velocity was assimilated rather than surface velocity, and with river discharge turned on. While this observation type is unrealistic from a practical standpoint (no practical method exists to measure depth-averaged currents synoptically), it serves to illustrate the role of baroclinic dynamics in reducing inversion skill. With depth-averaged currents, the RMSE (black lines in Fig. 9) was nearly always improved. The largest improvement was observed during neap tide, while improvement was less apparent during spring tide when baroclinic effects are smaller due to larger vertical mixing. The RMS difference between depth-averaged and surface velocities at the observation points ranged from 0.3 to $0.83 \mathrm{~m} \mathrm{~s}^{-1}$, with larger values occurring during ebb tide. Not shown in Fig. 9, depth-averaged currents could also recover bathymetry over a wider spatial 

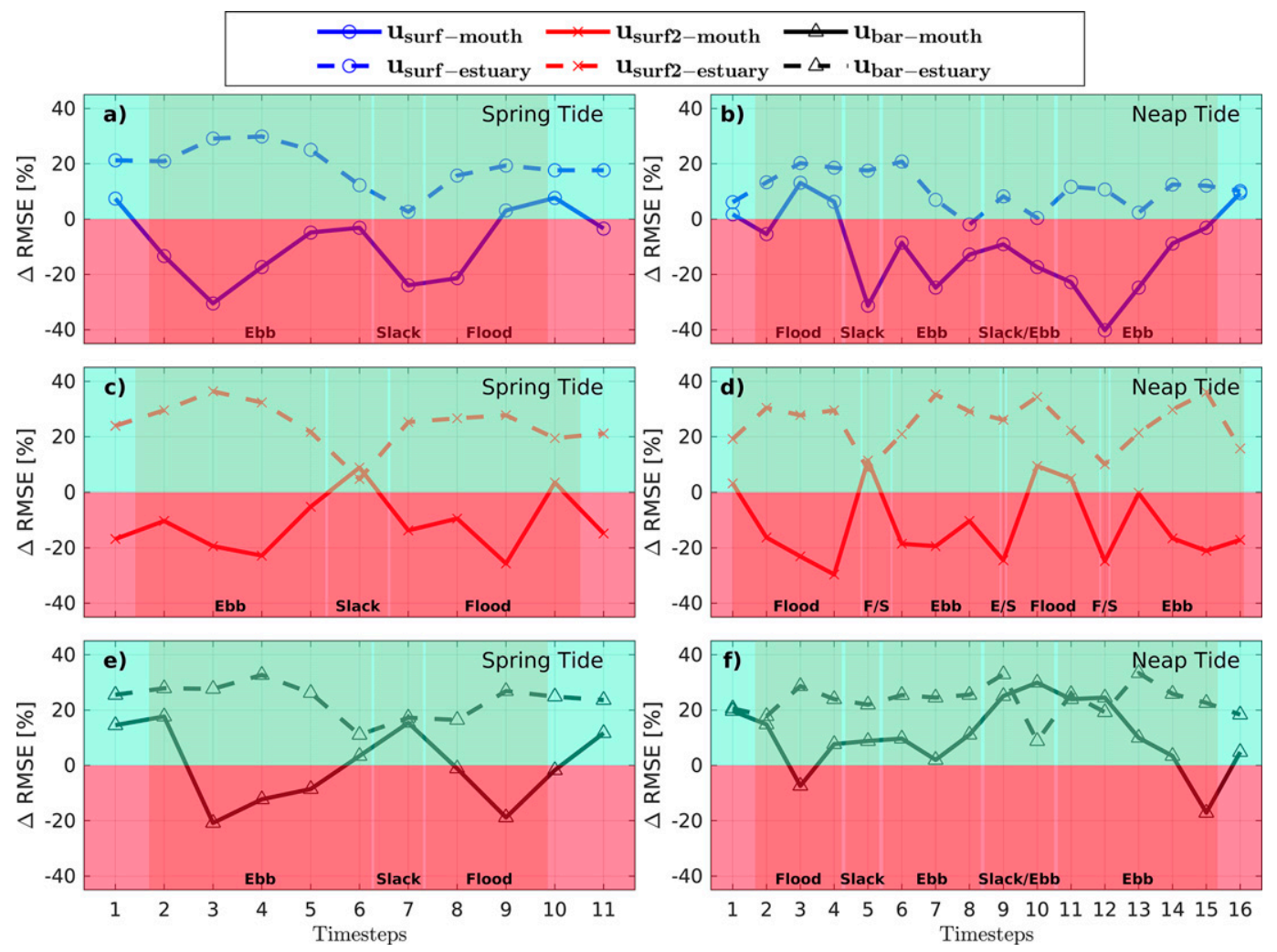

FIG. 10. Inverted bathymetry RMSE (as in Fig. 9) relative to prior RMSE (i.e., positive values indicate skillful inversion), for two spatial subdomains shown in Fig. 4: mouth (solid lines) and estuary (dashed lines). (a),(c),(e) Columns show results during spring tide and (b),(d),(f) results during neap tide. Rows show results for assimilation of (a),(b) surface currents; (c),(d) surface currents but with no river discharge in model; and (e),(f) depth-averaged currents.

area, including accurate predictions offshore of the river mouth where surface currents were insensitive to bathymetry (Fig. 7).

The above results address time dependence of model skill, showing modulations with tidal phase and between spring and neap tides. Spatial dependencies were investigated by splitting the observation array into the two subdomains shown in Fig. 4. Figure 10 shows RMSE versus time for the two regions, with dashed lines indicating the deeper-water region of the river mouth, and solid lines indicating the estuary. Since the two subdomains have different mean water depths and different prior RMSE values (3.51 and $8.92 \mathrm{~m}$ for mouth and estuary, respectively), results have been normalized to show the percent change in RMSE relative to the prior, with positive values indicating positive skill. Spring and neap time periods have been separated as in Fig. 9, and are shown in the lefthand and right-hand subfigures, respectively. Results are shown for all three scenarios considered previously-in order from top to bottom of the figure, they show inversion of surface currents with river discharge, surface currents without river discharge, and depth-averaged currents.

Several conclusions can be drawn from Fig. 10. A replication of Fig. 9 is observed for the estuary region (dashed lines), as regards to assimilating surface velocities in Figs. 10a-d; lower skill occurs during slack tide for the spring tidal stage, and during slack/ebb tide for neap tidal stages. The region of the river mouth (solid lines), on the other hand, has consistently negative skill, i.e., the corrections resulted in a bathymetry that was less accurate than the prior. Exceptions occur during weak ebb and slack tidal phases, in direct contrast to skill in the estuary (dashed lines) or for the overall domain (Fig. 9). Finally, use of barotropic velocities generally improves skill, including in the river mouth (solid lines), and especially for the neap tide period. Overall, the results suggest that the sensitivity of surface currents to bathymetry are minimal in the river mouth, consistent with adjoint sensitivities shown in Fig. 7. As water depth increases toward the outside of the estuary, surface current velocities evidently become decoupled from the seafloor geometry. Moghimi et al. (2016) drew a similar conclusion for depth inversion at the New River Inlet, North Carolina. In their study, surface currents did not provide any meaningful corrections in areas offshore of the inlet mouth. As can be seen in Figs. 10c and 10d, inclusion or exclusion of the river discharge did not impact this outcome, although some changes in tidal phasing can be observed. Use of depth-averaged currents did lead to an 
improvement, however, creating positive skill at the river mouth for some time periods.

Taken together, the results shown in Figs. 9 and 10 indicate inversion skill is maximized in locations/times with high flow, strong vertical mixing, and low river discharge. This would suggest that enhanced vertical stratification, associated with conditions of weak vertical mixing and/or river discharge, causes the surface current to become decoupled from bathymetry-as would be expected, for example, in a near-surface buoyant river plume or near-bottom salt wedge. Attempts to quantify these effects in greater detail were unsuccessful. For example, simple metrics based on local vertical velocity shear or buoyancy gradients did not directly correlate with inversion skill; we can only attribute these negative results to the complex 4D nature of the estuary. Nevertheless, the general trends shown by the inversion tests all support a conclusion that higher estuarine mixing, hence more barotropic flow, tended to correlate with higher inversion skill.

\section{Summary and conclusions}

An inversion scheme was applied to estimate bathymetry based on observations of surface currents in the mouth of the Columbia River (MCR), during a period of strong river discharge (spring freshet) which created conditions of a partially mixed estuary. The method was tested using a series of identical-twin tests, which compared hypothetical inversion scenarios spanning a range of tidal stages and river discharge levels. The types of variables inverted were surface current velocities $\mathbf{u}_{\text {surf }}$ and depth-averaged velocities $\mathbf{u}_{\text {bar }}$ collected as spatially distributed observations sampled at 1.5 -h intervals. Adjoint sensitivity analysis revealed how observations at different tidal stages and/or in different parts of the estuary provided more/less information on bathymetry, which led to a flowdependent inversion skill.

The inverse scheme was found to recover bathymetry with good accuracy, indicating that it successfully detects relationships between water depth and surface current. For example, Fig. 5 shows an inversion incorporating 11 observation times (with 1.5-h spacing), and including iterations to account for nonlinear effects. With each iteration, areas with high initial skill became reinforced, resulting in gradual improvements until convergence. The use of iterative inversion of distributed space-time observations resulted in higher skill than cases where fewer observations were used (e.g., single-time snapshots), or when iterations were not used. Much of the skill added by iterations came from a gradual refinement of deep channel-like features in the bathymetry, whose depth would otherwise be less accurately predicted by a noniterative method [as has been suggested in previous studies, e.g., Wilson and Özkan-Haller (2012) and Moghimi et al. (2016)]. The iterative method is able to achieve better results than a noniterative one (e.g., Landon et al. 2014) since it is less bound to the choice of initial prior bathymetry as a constraint.

Adjoint sensitivity analysis was used to determine times and locations where surface currents are most sensitive to bathymetry, i.e., when inversion is likely to be most successful.
For example, Fig. 7a shows adjoint sensitivity vectors associated with the inversion result in Fig. 8. It is apparent that the corrections made during inversion are concentrated on the areas with higher adjoint sensitivity, while regions of lower sensitivity received smaller corrections and hence had lower accuracy. The magnitude of bathymetry corrections also depends on the difference between prior and observed currents [( $\left.\mathbf{d}-\mathbf{M h}^{f}\right)$ in Eq. (4)]; for instance, corrections were smaller (hence lower accuracy) during slack tides when the difference $\left(\mathbf{d}-\mathbf{M h}^{f}\right)$ is small relative to either the observation noise level (defined by $R$ ), the variance in ensemble velocities, or both.

The example shown by Figs. $7 \mathrm{a}$ and 8 is also representative of results at other times. In general, surface currents are most sensitive to bathymetry in the narrow region of the river mouth, while surface currents offshore of the mouth (i.e., on the continental shelf) and far upstream have nearly no dependence on bathymetry. This spatial dependence was also supported by inversion test cases in which observations were restricted to certain parts of the study region (Fig. 10).

In contrast, observations of depth-averaged current did not have the same pattern of sensitivity as surface currents, and generally resulted in better inversions, suggesting an important role of baroclinicity. Similarly, conditions associated with higher estuary mixing tended to produce better inverse results: inversions during spring tide were more skillful than during neap tide, and inversions during low/zero river discharge more skillful than when river discharge was included. Hence, it appears baroclinic effects strongly affect the sensitivity of surface currents to bathymetry. Attempts to quantify inversion skill based on simple time-dependent metrics (e.g., bulk measures of vertical stratification) were not successful, however. Further studies are recommended to identify the specific dynamic effects that influence surface current sensitivity to bathymetry, perhaps by using idealized estuary simulations.

In summary, surface currents in large partially mixed estuaries can be inverted to retrieve estuary bathymetry. However, the relationship between the two variables is strongly flow dependent, and is not easily translated to simple inverse schemes. In particular, baroclinic dynamics appear to have a significant impact on inversion results-implying that any inversion scheme must accurately represent the 3D timedependent estuary dynamics. The space-time-varying sensitivity of surface currents to bathymetry also implies that, for maximum inverse skill, observations must be broadly distributed in space and time. Alternatively, observation arrays could incorporate variables other than surface current which may carry complimentary information (e.g., as found by Moghimi et al. 2016). Techniques from data assimilation provide the needed tools to tackle such problems, as they are designed to invert fully resolved ocean models based on arbitrarily distributed observations.

Acknowledgments. This work was supported by ONR Grant N00014-19-1-2218. We gratefully acknowledge Çiğdem Akan and Alexander Kurapov for their contributions to models used in this work, and for valuable discussions. Computational resources for this study were provided by grants 
from the DoD High Performance Computing Modernization Program at Navy DoD Supercomputing Resource Center and Air Force Research Laboratory; and by Extreme Science and Engineering Discovery Environment (XSEDE), which is supported by National Science Foundation grant number TG-OCE190003. Model inputs necessary for reproducing the calculations have been archived and are available upon request to the authors.

\section{REFERENCES}

Akan, Ç., S. Moghimi, H. T. Özkan-Haller, J. Osborne, and A. L. Kurapov, 2017: On the dynamics of the mouth of the Columbia River: Results from a three-dimensional fully coupled wave-current interaction model. J. Geophys. Res. Oceans, 122, 5218-5236, https://doi.org/10.1002/2016JC012307.

—, J. C. McWilliams, S. Moghimi, and H. T. Özkan-Haller, 2018: Frontal dynamics at the edge of the Columbia River plume. Ocean Modell., 122, 1-12, https://doi.org/10.1016/j. ocemod.2017.12.001.

Almeida, T. G., D. T. Walker, and A. M. Warnock, 2018: Estimating river bathymetry from surface velocity observations using variational inverse modeling. J. Atmos. Oceanic Technol., 35, 21-34, https://doi.org/10.1175/JTECH-D-17-0075.1.

Ardhuin, F., and Coauthors, 2018: Measuring currents, ice drift, and waves from space: The Sea surface KInematics Multiscale monitoring (SKIM) concept. Ocean Sci., 14, 337-354, https://doi.org/10.5194/os-14-337-2018.

Baptista, A. M., and Coauthors, 2015: Infrastructure for collaborative science and societal applications in the Columbia River estuary. Front. Earth Sci., 9, 659-682, https://doi.org/10.1007/ s11707-015-0540-5.

Chawla, A., D. A. Jay, A. M. Baptista, M. Wilkin, and C. Seaton, 2008: Seasonal variability and estuary-shelf interactions in circulation dynamics of a river-dominated estuary. Estuaries Coasts, 31, 269-288, https://doi.org/10.1007/s12237-007-9022-7.

Chung, H., J. Lee, and W. Y. Lee, 2021: A review: Marine biologging of animal behaviour and ocean environments. Ocean Sci. J., 56, 117-131, https://doi.org/10.1007/s12601-021-00015-1.

Egbert, G. D., and S. Y. Erofeeva, 2002: Efficient inverse modeling of barotropic ocean tides. J. Atmos. Oceanic Technol., 19, 183-204, https://doi.org/10.1175/1520-0426(2002)019<0183: EIMOBO $>2.0 . \mathrm{CO} ; 2$.

Evensen, G., 2009: Data Assimilation: The Ensemble Kalman Filter. Springer, 307 pp., https://doi.org/10.1007/978-3-642-037115 .

Fairall, C. W., E. F. Bradley, D. P. Rogers, J. B. Edson, and G. S. Young, 1996: Bulk parameterization of air-sea fluxes for Tropical Ocean-Global Atmosphere Coupled-Ocean Atmosphere Response Experiment. J. Geophys. Res., 101, 37473764, https://doi.org/10.1029/95JC03205.

Feddersen, F., R. T. Guza, and S. Elgar, 2004: Inverse modeling of one-dimensional setup and alongshore current in the nearshore. J. Phys. Oceanogr., 34, 920-933, https://doi.org/10.1175/ 1520-0485(2004)034<0920:IMOOSA > 2.0.CO;2.

Hamill, T. M., J. S. Whitaker, and C. Snyder, 2001: Distance-dependent filtering of background error covariance estimates in an ensemble Kalman filter. Mon. Wea. Rev., 129, 2776-2790, https://doi.org/10.1175/1520-0493(2001)129<2776:DDFOBE $>2$. $0 . \mathrm{CO} ; 2$.

Holman, R., and M. C. Haller, 2013: Remote sensing of the nearshore. Annu. Rev. Mar. Sci., 5, 95-113, https://doi.org/10.1146/ annurev-marine-121211-172408.
Honegger, D. A., M. C. Haller, and R. A. Holman, 2020: Highresolution bathymetry estimates via X-band marine radar: 2 . Effects of currents at tidal inlets. Coastal Eng., 156, 103626, https://doi.org/10.1016/j.coastaleng.2019.103626.

Honnorat, M., J. Monnier, and F. X. Le Dimet, 2009: Lagrangian data assimilation for river hydraulics simulations. Comput. Vis. Sci., 12, 235-246, https://doi.org/10.1007/s00791-008-0089-x.

Horstmann, J., S. Falchetti, C. Wackerman, S. Maresca, M. J. Caruso, and H. C. Graber, 2015: Tropical cyclone winds retrieved from C-band cross-polarized synthetic aperture radar. IEEE Trans. Geosci. Remote Sens., 53, 2887-2898, https://doi.org/10.1109/TGRS.2014.2366433.

Kalnay, E., 2002: Atmospheric Modeling, Data Assimilation and Predictability. Cambridge University Press, 368 pp., https:// doi.org/10.1017/CBO9780511802270.

— - and S. C. Yang, 2010: Accelerating the spin-up of ensemble Kalman filtering. Quart. J. Roy. Meteor. Soc., 136, 1644-1651, https://doi.org/10.1002/qj.652.

Kärnä, T., A. M. Baptista, J. E. Lopez, P. J. Turner, C. McNeil, and T. B. Sanford, 2015: Numerical modeling of circulation in high-energy estuaries: A Columbia River estuary benchmark. Ocean Modell., 88, 54-71, https://doi.org/10.1016/j. ocemod.2015.01.001.

Kurapov, A. L., and H. T. Özkan-Haller, 2013: Bathymetry correction using an adjoint component of a coupled nearshore wavecirculation model: Tests with synthetic velocity data. J. Geophys. Res. Oceans, 118, 4673-4688, https://doi.org/10.1002/jgrc.20306.

, D. Foley, P. T. Strub, G. D. Egbert, and J. S. Allen, 2011: Variational assimilation of satellite observations in a coastal ocean model off Oregon. J. Geophys. Res., 116, C05006, https://doi.org/10.1029/2010JC006909.

— , S. Y. Erofeeva, and E. Myers, 2017: Coastal sea level variability in the US West Coast Ocean Forecast System (WCOFS). Ocean Dyn., 67, 23-36, https://doi.org/10.1007/ s10236-016-1013-4

Landon, K. C., G. W. Wilson, H. T. Özkan-Haller, and J. H. MacMahan, 2014: Bathymetry estimation using drifter-based velocity measurements on the Kootenai River, Idaho. $J$. Atmos. Oceanic Technol., 31, 503-514, https://oi.org/10.1175/ JTECH-D-13-00123.1.

Moghimi, S., H. T. Özkan-Haller, G. W. Wilson, and A. Kurapov, 2016: Data assimilation for bathymetry estimation at a tidal inlet. J. Atmos. Oceanic Technol., 33, 2145-2163, https://doi. org/10.1175/JTECH-D-14-00188.1.

Nash, J. D., L. F. Kilcher, and J. N. Moum, 2009: Structure and composition of a strongly stratified, tidally pulsed river plume. J. Geophys. Res., 114, C00B12, https://doi.org/10.1029/ 2008JC005036.

Roquet, F., and Coauthors, 2017: Ocean observations using tagged animals. Oceanography, 30, 139-139, https://doi.org/10.5670/ oceanog.2017.235.

Simeonov, J. A., K. T. Holland, and S. P. Anderson, 2019: River discharge and bathymetry estimation from inversion of surface currents and water surface elevation observations. $J$. Atmos. Oceanic Technol., 36, 69-86, https://doi.org/10.1175/ JTECH-D-18-0055.1.

Stanford, J. A., F. R. Hauer, S. V. Gregory, and E. B. Snyder, 2005: Columbia River basin. Rivers of North America, A. C. Benke and C. E. Cushing, Eds., Elsevier, 590-653, https://doi. org/10.1016/B978-012088253-3/50016-X.

Thomson, J., 2012: Wave breaking dissipation observed with "swift" drifters. J. Atmos. Oceanic Technol., 29, 1866-1882, https://doi.org/10.1175/JTECH-D-12-00018.1. 
Warner, J. C., B. Armstrong, R. He, and J. B. Zambon, 2010: Development of a Coupled Ocean-Atmosphere-Wave-Sediment Transport (COAWST) modeling system. Ocean Modell., 35, 230-244, https://doi.org/10.1016/j.ocemod.2010.07.010.

Wilson, G. W., and H. T. Özkan-Haller, 2012: Ensemble-based data assimilation for estimation of river depths. J. Atmos. Oceanic Technol., 29, 1558-1568, https://doi.org/10.1175/JTECH-D-1200014.1.

,-- , and R. A. Holman, 2010: Data assimilation and bathymetric inversion in a two-dimensional horizontal surf zone model. J. Geophys. Res., 115, C12057, https://doi.org/10.1029/ 2010JC006286.

,,,--- M. C. Haller, D. A. Honegger, and C. C. Chickadel, 2014: Surf zone bathymetry and circulation predictions via data assimilation of remote sensing observations.
J. Geophys. Res. Oceans, 119, 1993-2016, https://doi.org/10. 1002/2013JC009213.

Zaron, E. D., 2017a: Recent developments in bottom topography mapping using inverse methods. Data Assimilation for Atmospheric, Oceanic and Hydrologic Applications (Vol. III), Springer International Publishing, 241-258, https://doi.org/10. 1007/978-3-319-43415-5_11.

- 2017b: Topographic and frictional controls on tides in the Sea of Okhotsk. Ocean Modell., 117, 1-11, https://doi.org/10. 1016/j.ocemod.2017.06.011.

- M. A. Pradal, P. D. Miller, A. F. Blumberg, N. Georgas, W. Li, and J. M. Cornuelle, 2011: Bottom topography mapping via nonlinear data assimilation. J. Atmos. Oceanic Technol., 28, 1606-1623, https://doi.org/10.1175/JTECH-D-11-00070.1. 